\title{
Bronchial Clearance Physiotherapy in Pediatrics. A Controlled, Randomized, Multicenter Study of the Short-Term Effects on Respiration during Outpatient Care for Infants with Acute Bronchiolitis
}
S. Sebban ${ }^{1}$
D. Evenou ${ }^{2}$
C. Jung 3,4
C. Fausser ${ }^{1}$
S. Durand 5
M. Bibal ${ }^{5} \quad$ V. Geninasca 6
M. Saux ${ }^{6}$

J.C. Jeulin ${ }^{7}$

${ }^{1}$ Department of Physiotherapy, Association des Réseaux Bronchiolite. Teaching Hospital (CHU) Robert-Debré-APHP, Paris, France

2 Department of Physiotherapy, Association des Réseaux Bronchiolite, Teaching Hospital (CHU) Robert-Debré- APHP, Paris, France

${ }^{3}$ Department of Paediatrics, Clinical Research Centre, Centre Hospitalier Intercommunal de Créteil, Créteil, France

${ }^{4}$ Department of Massage Therapy/Physiotherapy, Paris Pubic Hospitals Group (APHP), Paris, France

${ }^{5}$ Department of Massage Therapy/Physiotherapy, Réseau bronchiolite lle de France

${ }^{6}$ Department of Massage Therapy/Physiotherapy, Réseau

Bronchiolite lle de France

${ }^{7}$ Department of Massage Therapy/Physiotherapy, Réseau

Bronchiolite, France

J Child Sci 2021;11:e1.

\section{Retraction}

Concerning the above article (J Child Sci 2021; 11(01): e155e162; doi: 10.1055/s-0041-1731304), the Editors have been made aware of a significant overlap with another article "Symptomatic Effects of Chest Physiotherapy with Increased Exhalation

Address for correspondence D. Evenou, MSc, Department of Physiotherapy, Association des Réseaux Bronchiolite, Teaching Hospital (CHU) Robert-Debré- APHP, 48 Bd Sérurier, 75019 Paris, France (e-mail: devenou@orange.fr).

Technique in Outpatient Care for Infant Bronchiolitis: A Multicentre, Randomised, Controlled Study" by Sebban et al published in Journal of Clinical Research and Medicine [2019;2(04)].

For this reason, the Editorial Board gives the notice that the article is retracted by the Editors in accordance with COPE guidelines. 